\title{
Abordagem de usuários de crack na Atenção Primária à Saúde: uma revisão sistemática
}

\author{
Approach to crack users in primary care: a systematic review
}

Abordaje de usuarios de crack en la Atención Primaria a la Salud: una revisión sistemática

Leda Chaves Dias. Serviço de Saúde Comunitária do Grupo Hospitalar Conceição (SSC-GHC). Porto Alegre, RS, Brasil. leda.curra@terra.com.br (Autora correspondente)

Erno Harzheim. Universidade Federal do Rio Grande do Sul (UFRGS). Porto Alegre, RS, Brasil. ernoharz@ terra.com.br

Caren Bavaresco. Hospital Nossa Senhora da Conceição. Porto Alegre, RS, Brasil. c_bavaresco@yahoo.com.br

Marcelo Rodrigues Gonçalves. Universidade Federal do Rio Grande do Sul (UFRGS). Porto Alegre, RS, Brasil. marcelorog@gmail.com

Mariana Dias Curra. Pontifícia Universidade Católica do Rio Grande do Sul (PUC-RS). Porto Alegre, RS, Brasil. mdiascurra@gmail.com

\section{Resumo}

Objetivo: identificar abordagens efetivas para o manejo de adultos usuários de crack no contexto da Atenção Primária à Saúde (APS). Métodos: revisão sistemática realizada nas principais bases de dados eletrônicos e estudos adicionais, que incluiu os estudos sobre "crack" ou "crack/cocaína" no contexto da pesquisa, publicados nos últimos dez anos. A avaliação de evidências foi realizada por dois pesquisadores independentes, por meio de instrumentos distintos, um para estudos observacionais, outro para os de intervenção. Resultados: foram incluídos na análise de evidências 16 estudos identificados a partir de 2017 referências. O tipo de delineamentos nas investigações incluiu estudos observacionais (6) e estudos de intervenção (10). Dois artigos observacionais apresentaram boa qualidade de evidência, e a melhor evidência encontrada entre os artigos de intervenção foi de nível moderado (um artigo). Conclusão: a revisão sistemática não obteve artigos que apresentassem evidências de alta qualidade para serem orientadas ao contexto da APS, pois nenhum estudo avaliando as atribuições da APS ou especificando o manejo dos profissionais neste nível foi encontrado. Permanecem as evidências geradas no período anterior ao estipulado pela revisão.

\section{Abstract}

Objective: to identify effective approaches for the management of adult crack users in the context of primary health care. Methods: we conducted a systematic review of major electronic databases and additional studies. All of the studies included the theme of "crack" or "crack/cocaine" in the research context, and had been published in the last 10 years. The evidence evaluation was performed by two independent researchers, using different instruments for observational compared to interventional studies. Results: sixteen out of 2,017 studies were included for evidence analysis, six of which were observational, while 10 were intervention studies. Two observational papers presented evidence of good quality and only one interventional article had moderate quality evidence. Conclusion: this systematic review did not obtain high quality evidence that could guide the context of primary health care. Evidence that was generated before this review remains as a reference.
Palavras-chave: Atenção Primária à Saúde Cocaína Crack Revisão

Keywords: Primary Health Care Crack Cocaine Review
Como citar: Dias LC, Harzheim E, Bavaresco C, Gonçalves MR, Curra MD. Abordagem de usuários de crack na Atenção Primária à Saúde: uma revisão sistemática. Rev Bras Med Fam Comunidade. 2015;10(36):1-14. http://dx.doi.org/10.5712/rbmfc10(36)911
Fonte de financiamento: declaram não haver. Parecer CEP: não se aplica. Conflito de interesses: declaram não haver. Procedência e revisão por pares: revisado por pares. Recebido em: 30/03/2014. Aprovado em: 03/08/2015. 


\section{Resumen}

Objetivo: identificar abordajes efectivos para el manejo de adultos usuarios de crack en el contexto de la APS. Metodología: revisión sistemática realizada en las principales bases de datos electrónicos y estudios adicionales incluyendo estudios sobre "crack" o "crack/cocaína" en el contexto de esta investigación, publicados en los últimos diez años. La evaluación de evidencias se realizó por dos investigadores independientes utilizando dos instrumentos distintos, uno para estudios observacionales y otro para los de intervención. Resultados: se incluyeron en el análisis de evidencias 16 estudios identificados a partir de 2.017 referencias. El tipo de delineamiento de las investigaciones incluyó estudios observacionales (6) y estudios de intervención (10). Dos artículos observacionales presentaron buena calidad de evidencia, y la mejor evidencia encontrada entre los artículos de intervención fue de nivel moderado (un artículo). Conclusión: la revisión sistemática no obtuvo artículos que presentaron evidencias de alta calidad orientadas al contexto de la APS, pues no se encontró ningún estudio evaluando las atribuciones de la APS o especificando el manejo de los profesionales en este nivel de atención. Permanecen las evidencias generadas en el período anterior al estipulado por la revisión.
Palabras clave:

Atención Primaria de Salud

Cocaína Crack

Revisión

\section{Introdução}

Nos últimos anos, temos visto uma retomada da discussão em torno do uso e abuso de drogas, principalmente da mais emergente, o "crack", uma forma de cocaína fumada que, neste formato, permite uma disseminação maciça da substância para o cérebro, obtendo efeitos mais estimulantes, muito mais prazerosos e com início de ação muito mais rápido. ${ }^{1}$

A droga é reconhecida por seu elevado potencial dependógeno, e a estimativa inicial da OMS (Organização Mundial da Saúde) para o Brasil, segundo o Conselho Federal de Medicina (CFM) e o Ministério da Saúde ${ }^{2}$ era de que existissem $3 \%$ de usuários envolvidos com cocaína e crack; entretanto, dados da última pesquisa realizada pela Fiocruz e divulgada em setembro deste ano revelam que os usuários regulares de crack e/ou de formas similares de cocaína fumada (pasta-base, merla e oxi) somam 370 mil pessoas nas 26 capitais brasileiras e no Distrito Federal. ${ }^{3}$ Embora os números tenham sido redimensionados com a pesquisa, persistem índices alarmantes, concentrando os holofotes em torno deste fenômeno. Surpreendentemente, apesar das ações políticas estarem estruturadas em três eixos (cuidado, autoridade e prevenção) o cuidado e a prevenção, que incluem ampliação e qualificação da rede de saúde voltada aos usuários, ${ }^{4}$ não têm sido inseridos como parte integrante de ação a Atenção Primária à Saúde (APS), ou, pelo menos, suas atribuições não estão dispostas de forma sistematizada e/ou clara.

A magnitude da situação gera extrema preocupação, que, por vezes, é ampliada pela divulgação dos meios de comunicação de maneira exploratória, o que repercute em respostas radicais ou focais. Os transtornos relacionados ao uso de substâncias são frequentes em todos os grupos demográficos e são comuns nos ambientes da APS. Trata-se de doença crônica que requer uma estratégia de ação por um tempo prolongado; logo, a APS, que provê a ênfase nas relações longitudinais e preventivas, pode ser o local ideal para o manejo adequado dessa condição em algum momento do cuidado.

Estudos da Associação Brasileira de Psiquiatria ${ }^{5}$ referem que os usuários de cocaína e crack têm muita dificuldade na busca de tratamento especializado por não reconhecerem que estão com o problema e por todo o processo de clandestinidade que envolve a drogadição. Além disto, muitas vezes, os serviços disponíveis não oferecem abordagens ajustadas às suas necessidades.

O objetivo deste artigo foi avaliar a evidência disponível, nos últimos dez anos, sobre abordagens efetivas para o manejo de adultos usuários de crack no contexto da APS. 


\section{Métodos}

\section{Definições Gerais}

Foi conduzida uma revisão sistemática de literatura sobre a abordagem de usuários de crack na APS. A estratégia de busca utilizada baseou-se em uma pergunta estruturada na forma P I C O (das iniciais "Paciente", "Intervenção", "Controle" e "Outcome" - resultado). ${ }^{6}$ A pergunta desenvolvida foi: "Quais abordagens são efetivas no manejo de adultos usuários de crack no contexto da Atenção Primária à Saúde?".

\section{Busca e Identificação de Estudos}

Em um formato "piloto", como tentativa inicial de identificação de estudos, foram testadas várias terminologias que incluíam todos os termos que especificassem o âmbito da pesquisa. Entretanto, a associação de termos referentes à Atenção Primária à Saúde, Atenção Básica à Saúde ou terminologia semelhante limitava o aporte de estudos, chegando a zerar o número de artigos em algumas bases de dados. Consequentemente, optamos por ampliar as buscas, de maneira que não incluíssem, necessariamente, estes termos e decidimos revisar maior número de bases de dados.

Os critérios de busca e identificação dos estudos foram: 1) Período de publicação: ser de 2003 a 2013; 2) Língua: Portuguesa, Espanhola ou Inglesa; 3) População: adulta, acima de 19 anos; e 4) Delineamento: inicialmente, foram incluídos todos os tipos de estudos referentes ao tema crack/crack-cocaína.

O período de busca da literatura ocorreu de 10 de janeiro a 19 de maio de 2013. Foram utilizadas as seguintes bases de dados: PubMed, EMBASE, CRD (NHS - National Institute for Health Research), LILACS, Google Acadêmico, TRIPDATABASE, NICE, National Guideline Clearinghouse e Cochrane. Estudos adicionais foram, também, pesquisados no site da Biblioteca Digital Brasileira de Teses e Dissertações (http://bdtd.ibict.br/), assim como foram examinadas as bibliografias dos artigos de sites dos artigos originais, do livro identificado na busca dos estudos, nas revisões sistemáticas e nos protocolos identificados.

A sintaxe da estratégia de busca foi adaptada ao formato de cada base de dados. Como ilustração, a sintaxe usada no PubMed foi: Busca - ("Outcome Assessment (Health Care)"[Mesh]) AND ("Cocaine"[Mesh] OR "Cocaine - Related Disorders"[Mesh] OR "Crack Cocaine"[Mesh]) Filtros: Filters activated: published in the last 10 years, Humans, English, Portuguese, Spanish, Adult: 19+ years.

\section{Seleção de estudos}

Os estudos identificados com a estratégia de busca tiveram origem em todas as bases de dados referidas e bibliografias extras dos artigos originais, livros, protocolos e artigos citados nos sites dos artigos originais. A partir do total identificado, foi realizada uma primeira seleção, em que foram removidas todas as publicações em que, pelo título do estudo, claramente, fossem detectadas outras temáticas, ou que referissem ser com população de crianças e/ou adolescentes ou estivessem duplicadas.

Uma segunda seleção foi realizada e todos os artigos que não incluíssem a temática do "crack" ou "crack/cocaína" no contexto da pesquisa foram eliminados. Esta avaliação ocorreu após a leitura de todos os resumos dos artigos. 


\section{Procedimentos de Inclusão}

Todos os estudos incluídos nesta revisão tiveram como objetivo principal ou secundário uma abordagem relativa ao manejo do usuário de crack.

\section{Extração de dados dos artigos incluídos}

A etapa seguinte foi a leitura integral das referências selecionadas e incluídas, e a organização de um quadro descritivo destes artigos (Quadro 1).

Quadro 1. Descrição dos artigos observacionais sobre manejo do crack incluídos na revisão sitemática, Porto Alegre, 2013.

\begin{tabular}{|c|c|c|c|c|c|c|}
\hline Ref & Local/Ano & Delineamento & População & Objetivo & Resultados & $\mathbf{N}$ \\
\hline 23 & $\begin{array}{l}\text { Porto Alegre, } \\
\text { RS, Brasil } \\
2009\end{array}$ & Transversal & $\begin{array}{c}\text { Pacientes } \\
\text { internados para } \\
\text { desintoxicação }\end{array}$ & $\begin{array}{l}\text { Analisar as estratégias } \\
\text { de coping para o manejo } \\
\text { da fissura em dependen- } \\
\text { tes de crack internados. }\end{array}$ & $\begin{array}{c}\text { Foram encontradas correlações positivas } \\
\text { de intensidade moderada entre a motivação } \\
\text { para a interrupção do uso do crack e as } \\
\text { estratégias Resolução de Problemas e } \\
\text { Fuga-Esquiva, e correlações também } \\
\text { positivas, mas de fraca intensidade com } \\
\text { as estratégias Autocontrole e Reavaliação } \\
\text { Positiva. }\end{array}$ & 35 \\
\hline 36 & $\begin{array}{l}\text { Carolina do } \\
\text { Norte, EUA } \\
2000-2002\end{array}$ & Transversal & $\begin{array}{l}\text { Usuários de crack } \\
\text { de rua negros }\end{array}$ & $\begin{array}{l}\text { Comparar dois grupos de } \\
\text { usuários de crack e ava- } \\
\text { liar quais os fatores estão } \\
\text { associados à motivação } \\
\text { para o tratamento. }\end{array}$ & $\begin{array}{l}\text { As muitas necessidades não satisfeitas } \\
\text { relatadas por usuários de crack motivados } \\
\text { para o tratamento sugerem que a entrada } \\
\text { ao tratamento e retenção pode ser } \\
\text { facilitada pelo pré-tratamento e tratamento } \\
\text { auxiliar. }\end{array}$ & 345 \\
\hline 27 & $\begin{array}{l}\text { Londres, } \\
\text { Inglaterra } \\
2003\end{array}$ & Coorte & $\begin{array}{l}\text { Usuários de } \\
\text { comunidades } \\
\text { terapêuticas de } \\
\text { crise }\end{array}$ & $\begin{array}{l}\text { Melhorar a compreensão } \\
\text { sobre o uso de drogas e } \\
\text { identificar formas efica- } \\
\text { zes de intervenção. }\end{array}$ & $\begin{array}{l}\text { Os usuários fazem uso de polidrogas; } \\
\text { as minorias étnicas usam menos drogas } \\
\text { injetáveis; média de gasto/dia de } 100 \\
\text { pounds; a maioria dos entrevistados } \\
\text { financia sua utilização por meio do crime. }\end{array}$ & 100 \\
\hline 26 & $\begin{array}{l}\text { São Paulo, } \\
\text { Brasil } \\
2006\end{array}$ & Série de casos & $\begin{array}{c}\text { Usuários } \\
\text { admitidos para } \\
\text { desintoxicação }\end{array}$ & $\begin{array}{l}\text { Analisar a evolução do } \\
\text { consumo de droga entre } \\
\text { usuários de crack com } \\
\text { histórico de tratamento. }\end{array}$ & $\begin{array}{l}\text { Foram identificados três grupos com } \\
\text { trajetórias distintas de consumo pós-alta. } \\
\text { Comportamento seguro com uso de } \\
\text { preservativo foi identificado como fator } \\
\text { relacionado ao grupo de abstinentes } \\
\text { estáveis ( } p=0,001 \text { ). Teste HIV positivo } \\
\text { na internação ( } p=0,046) \text {; consumo de } \\
\text { cocaína aspirada no último ano ( } p=0,001 \text { ) } \\
\text { e tempo de uso de cocaína aspirada na } \\
\text { vida (mais de } 132 \text { meses) ( } p=0,000 \text { ) foram } \\
\text { fatores relacionados a uso de longo tempo. } \\
\text { Uso pregresso de cocaína endovenosa } \\
\text { aumentou } 2,5 \text { vezes as chances de óbito } \\
\text { em } 12 \text { anos ( } p=0,031 \text { ) (IC95\%: } 1,08 ; 5,79) \text {. }\end{array}$ & 131 \\
\hline 38 & $\begin{array}{l}\text { Porto Alegre, } \\
\text { Brasil } \\
2006 / 2007\end{array}$ & Série de casos & $\begin{array}{l}\text { Jovens usuários } \\
\text { de crack } \\
\text { que buscam } \\
\text { aconselhamento } \\
\text { telefônico }\end{array}$ & $\begin{array}{c}\text { Buscou avaliar a } \\
\text { utilização da Intervenção } \\
\text { Breve Motivacional } \\
\text { (IBM) em um serviço } \\
\text { de aconselhamento } \\
\text { telefônico. }\end{array}$ & $\begin{array}{l}\text { A partir dos resultados obtidos, pode- } \\
\text { se sugerir que a IBM associada ao } \\
\text { aconselhamento telefônico representa uma } \\
\text { alternativa de tratamento importante na } \\
\text { cessação do uso de crack. }\end{array}$ & 40 \\
\hline
\end{tabular}


Continuação Quadro 1.

\begin{tabular}{|c|c|c|c|c|c|c|}
\hline 37 & $\begin{array}{l}\text { Porto Alegre, } \\
\text { Brasil } \\
\text {----- }\end{array}$ & $\begin{array}{l}\text { Ensaio clínico do } \\
\text { tipo quase-ex- } \\
\text { perimental, tipo } \\
\text { antes e depois }\end{array}$ & $\begin{array}{l}\text { Usuários de } \\
\text { Unidade de inter- } \\
\text { nação }\end{array}$ & $\begin{array}{l}\text { Avaliar a efetividade dos } \\
\text { jogos cooperativos no } \\
\text { manejo da fissura. }\end{array}$ & $\begin{array}{l}\text { Foi encontrada diferença significativa no } \\
\text { craving e sintomas de ansiedade a partir } \\
\text { dos jogos cooperativos }(p<0,001) \text {, porém } \\
\text { não houve alteração na motivação para } \\
\text { mudança do comportamento aditivo. }\end{array}$ & 30 \\
\hline 35 & $\begin{array}{l}\text { Porto Alegre, } \\
\text { Brasil } \\
2008\end{array}$ & $\begin{array}{l}\text { Ensaio clínico } \\
\text { quase-experi- } \\
\text { mental tipo antes } \\
\text { e depois }\end{array}$ & Usuários de crack & $\begin{array}{l}\text { Verificar a efetividade } \\
\text { do relaxamento } \\
\text { respiratório no manejo } \\
\text { do craving e dos } \\
\text { sintomas de ansiedade } \\
\text { em dependentes } \\
\text { de crack internados } \\
\text { para tratamento em } \\
\text { uma unidade de } \\
\text { desintoxicação. }\end{array}$ & $\begin{array}{l}\text { Os resultados desta pesquisa } \\
\text { demonstraram uma redução dos } \\
\text { escores do CCQB, da EAV e do BAI pelo } \\
\text { relaxamento respiratório em uma amostra } \\
\text { cujo perfi I corresponde ao padrão geral } \\
\text { dos usuários de crack. }\end{array}$ & 32 \\
\hline 25 & $\begin{array}{l}\text { Denver, } \\
\text { Colorado, } \\
\text { EUA } \\
2004 / 2006\end{array}$ & $\begin{array}{l}\text { Ensaio clínico do } \\
\text { tipo quase-ex- } \\
\text { perimental, tipo } \\
\text { antes e depois }\end{array}$ & $\begin{array}{l}\text { Mulheres usuárias } \\
\text { na comunidade }\end{array}$ & $\begin{array}{l}\text { Avaliar a intervenção } \\
\text { de manejo de caso em } \\
\text { mulheres que usam } \\
\text { crack. }\end{array}$ & $\begin{array}{l}\text { Os resultados indicam que o "serviço de } \\
\text { manejo de casos" é útil para ajudar as } \\
\text { mulheres que usam crack melhorar as } \\
\text { suas vidas. }\end{array}$ & 149 \\
\hline 32 & $\begin{array}{l}\text { Tennessee, } \\
\text { EUA }\end{array}$ & $\begin{array}{l}\text { Ensaio clínico do } \\
\text { tipo quase-ex- } \\
\text { perimental, tipo } \\
\text { antes e depois }\end{array}$ & $\begin{array}{l}\text { Mulheres negras } \\
\text { usuárias de crack }\end{array}$ & $\begin{array}{l}\text { Avaliar um programa de } \\
\text { tratamento para usuárias } \\
\text { que estão em risco de } \\
\text { contrair AIDS. }\end{array}$ & $\begin{array}{l}\text { O programa ajuda a reduzir o } \\
\text { comportamento de risco para HIV e } \\
\text { diminuição do uso da substância. }\end{array}$ & 207 \\
\hline 34 & $\begin{array}{l}\text { Carolina do } \\
\text { Norte, EUA } \\
\text {----- }\end{array}$ & ECR & $\begin{array}{l}\text { Usuários negros } \\
\text { recrutados }\end{array}$ & $\begin{array}{l}\text { Examinar os efeitos } \\
\text { de uma intervenção } \\
\text { pré-tratamento projetado } \\
\text { para melhorar motivação } \\
\text { para o tratamento, dimi- } \\
\text { nuir o uso de crack. }\end{array}$ & $\begin{array}{l}\text { Aos } 3 \text { meses de follow-up, ambos os } \\
\text { grupos reduziram significativamente } \\
\text { seu uso de crack, mas os participantes } \\
\text { do grupo de intervenção foram mais } \\
\text { propensos a ter iniciado o tratamento. }\end{array}$ & 443 \\
\hline 28 & $\begin{array}{l}\text { Nova York, } \\
\text { EUA } \\
\text {--- }\end{array}$ & $\begin{array}{c}\text { EC não } \\
\text { randomizado }\end{array}$ & $\begin{array}{l}\text { Usuários } \\
\text { hospitalizados e } \\
\text { ambulatoriais }\end{array}$ & $\begin{array}{l}\text { Examinar os efeitos } \\
\text { da Gabapentina na } \\
\text { manutenção do controle } \\
\text { de uso da droga. }\end{array}$ & $\begin{array}{l}\text { Gabapentina não reduz efeitos positivos do } \\
\qquad \operatorname{crack}(p<0,05)\end{array}$ & 12 \\
\hline 24 & $\begin{array}{l}\text { Kansas City, } \\
\text { Missouri, } \\
\text { EUA } \\
\text {------ }\end{array}$ & ECR & $\begin{array}{l}\text { Usuários admiti- } \\
\text { dos em um centro } \\
\text { de tratamento }\end{array}$ & $\begin{array}{l}\text { Comparar os efeitos } \\
\text { da desipramine ou } \\
\text { carbamazepina } \\
\text { ao placebo em um } \\
\text { programa intensivo para } \\
\text { abusadores de crack. }\end{array}$ & $\begin{array}{c}\text { Há uma possibilidade da desipramina ter } \\
\text { algum papel no tratamento do abuso de } \\
\text { crack. }\end{array}$ & 146 \\
\hline 33 & $\begin{array}{l}\text { Atlanta, EUA } \\
1998-2001\end{array}$ & ECR & $\begin{array}{l}\text { Mulheres negras } \\
\text { usuárias de rua }\end{array}$ & $\begin{array}{c}\text { Avaliar a eficácia de uma } \\
\text { intervenção para redução } \\
\text { de risco em mulheres } \\
\text { usuárias de crack } \\
\text { soronegativas para HIV. }\end{array}$ & $\begin{array}{l}\text { O programa de redução de danos foi es- } \\
\text { tatisticamente significante para reduzir o } \\
\text { consumo de crack }(p<0.05) \text {. }\end{array}$ & 265 \\
\hline 30 & EUA & ECR & $\begin{array}{l}\text { Adultos usuários } \\
\text { de crack e HIV } \\
\text { positivos }\end{array}$ & $\begin{array}{c}\text { Testar a viabilidade } \\
\text { e a eficácia de } \\
\text { duas intervenções } \\
\text { baseadas no modelo } \\
\text { de competências de } \\
\text { informação-motivação } \\
\text { comportamental para } \\
\text { melhorar a adesão } \\
\text { HAART (tratamento } \\
\text { para o HIV) e reduzir } \\
\text { problemas. }\end{array}$ & $\begin{array}{l}\text { Aumentos significativos na adesão e } \\
\text { reduções de ASI drogas Scores Composite } \\
\text { ocorreu em ambas as condições, de } 3 \\
\text { meses e foram mantidas a } 6 \text { meses, o } \\
\text { que representa médias tamanhos de } \\
\text { efeito. Nenhuma diferença entre os grupos } \\
\text { foram observadas. Nenhuma mudança foi } \\
\text { observada no VL ou grupo. Credibilidade } \\
\text { do tratamento, retenção e satisfação eram } \\
\text { altas e não pela condição diferente. }\end{array}$ & 42 \\
\hline 29 & $\begin{array}{l}\text { Roterdam, } \\
\text { Holanda } \\
2000-2001\end{array}$ & ECR & $\begin{array}{l}\text { Consumidores } \\
\text { crônicos de crack }\end{array}$ & $\begin{array}{l}\text { Avaliar a eficácia de um } \\
\text { programa extramuros } \\
\text { quando comparado a um } \\
\text { programa padrão. }\end{array}$ & $\begin{array}{l}\text { Os usuários tratados no programa } \\
\text { extramuros tiveram melhora significativa na } \\
\text { saúde física, condições gerais de vida, e } \\
\text { estado psiquiátrico, mas nenhuma melhora } \\
\text { no emprego, no abuso da substância e na } \\
\text { condição legal. }\end{array}$ & 124 \\
\hline
\end{tabular}


Avaliação da qualidade metodológica

Para avaliar a qualidade metodológica dos estudos, participaram dois revisores (CB e EH) independentes, e, em caso de discordância entre eles, usou-se o julgamento de uma terceira pessoa (MG). A avaliação de qualidade foi realizada por unidade de estudo.

Para os estudos observacionais, foi aplicado um instrumento de avaliação produzido pela Universidade de Wales, ${ }^{7}$ que é um protocolo que permite avaliar problemas de saúde que ainda não foram muito estudados por estudos controlados randomizados, sendo a evidência de alta qualidade procurada dentro de outros tipos de evidências, o que é particularmente relevante dentro dos cuidados primários. Este instrumento é constituído por treze critérios de qualidade relacionados aos estudos. A avaliação foi qualitativa, de maneira dicotômica, com qualificação de "sim", "não" ou "não se aplica", de acordo com a presença ou ausência do critério avaliado. Considerou-se que a soma da pontuação acima de dois terços de presença de "sim", equivalendo à pontuação maior que 8, expressavam boa evidência científica a favor do estudo. A escolha do tercil superior como ponto de corte foi definida pelos autores buscando-se uma correlação com outros escores de avaliação de evidências.

Os estudos de intervenção foram avaliados de acordo com o sistema GRADE Working Group, ${ }^{8}$ ou seja, a qualidade da evidência foi classificada em: A - alta qualidade; B - moderada qualidade; C - baixa qualidade; D - muito baixa qualidade. Os instrumentos foram aplicados individualmente por cada revisor e, após, comparados para a avaliação de qualidade. No caso de discordância de avaliação entre os pesquisadores, o terceiro revisor definia a avaliação mediante o resgate dos artigos originais.

Foi desenvolvida uma revisão sistemática com enfoque mais qualitativo e metodológico. Não foi possível desenvolver uma meta-análise devido à heterogeneidade dos estudos.

\section{Resultados}

Foram identificadas 2017 referências decorrentes da busca em diversas bases de dados bibliográficos.

A primeira etapa de seleção eliminou 1927 referências, resultando em 90 estudos selecionados. A segunda etapa de seleção excluiu 59 artigos e elegeu 31 estudos.

Os 31 estudos eleitos foram separados, com texto completo, para avaliar a inclusão no estudo. Desses 31 estudos, 15 não foram incluídos ${ }^{5,9-22}$ por serem: revisão sistemática, diretriz clínica, revisão narrativa, estudo de validação de instrumento, estudos qualitativos, livro e estudo de caso. Então, os 16 estudos incluídos ${ }^{23-38}$ foram distinguidos quanto aos seus delineamentos e classificados em dois grupos, os observacionais e os de intervenção.

Os resultados da estratégia de busca e seleção encontram-se dispostos na Figura 1.

O Quadro 1 apresenta a descrição sumária dos 16 estudos incluídos nesta revisão sistemática e os principais resultados. O período de publicação solicitado pela busca de referências foi de 2003 a 2013, entretanto, três deles foram realizados em períodos anteriores (1998-2001, 2000-2001 e 2000-2002) e, em seis, não constava o período de realização. Os países que contribuíram com estudos foram: EUA (8), 


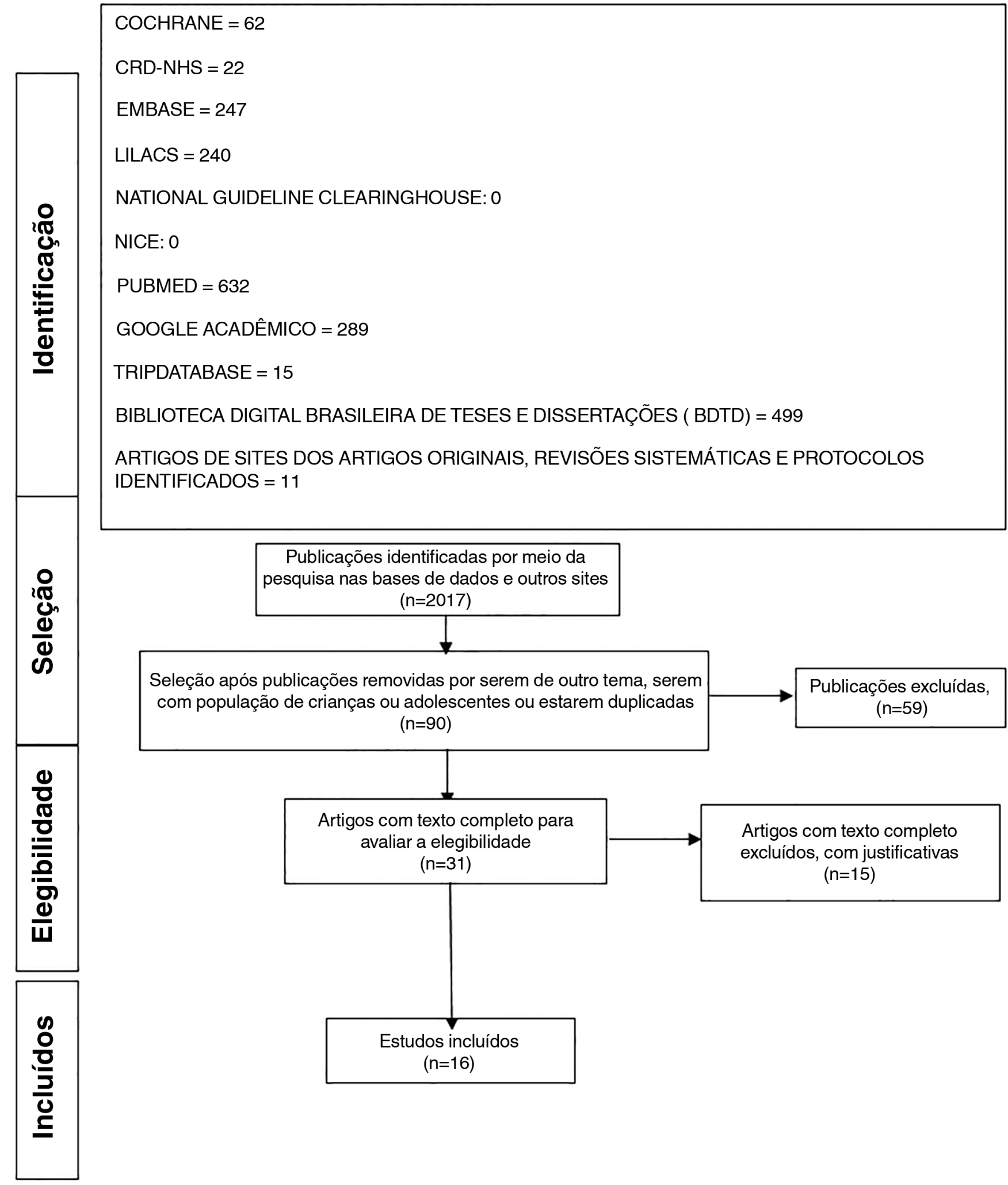

Figura 1. Resultados da estratégia de busca e seleção dos estudos. ${ }^{39}$ 
Brasil (5), Inglaterra (2) e Holanda (1). Os tipos de delineamento nas investigações incluíram estudos observacionais (dois transversais, duas coortes e duas séries de casos) e estudos de intervenção (quatro ensaios clínicos (EC)) tipo quase experimental tipo antes e depois, um ensaio clínico não randomizado e cinco ensaios clínicos randomizados (ECR)). Quanto à população estudada, referiam, basicamente, quatro origens: os internados (4), os usuários de rua (2), os usuários de comunidade terapêutica (3) e aqueles que foram recrutados (7).

A Tabela 1 mostra a avaliação metodológica dos seis estudos observacionais pelo instrumento de Wales, que apresentou dois artigos de boa qualidade, com pontuação superior a 8. Segundo este instrumento, todos os estudos eram relevantes e tinham clareza de objetivos; apenas um tinha metodologia adequada e foi considerada a possibilidade de vieses; a população mostrou-se inadequada em quatro de seis estudos; a análise estatística apresentada foi bem empregada e bem escolhida em metade dos estudos, assim como os resultados que eram importantes. Nenhum dos estudos apresentava informação sobre custos.

A Tabela 2 apresenta a avaliação metodológica dos 10 estudos com classificação do nível de evidência segundo o Sistema GRADE (Qualidade da evidência - A: alta; B: moderada; C: baixa; e D: muito baixa). Assim, apenas um estudo foi classificado com qualidade de evidência moderada; cinco receberam a avaliação de baixa qualidade de evidência, e quatro foram avaliados como qualidade muito baixa de evidência.

Em três estudos, ocorreu discordância de avaliação entre EH e CB, um observacional e dois de intervenção, que foram consensuados pelo terceiro avaliador (MG).

\section{Discussão}

O objetivo deste artigo foi revisar os estudos sobre abordagens efetivas para serviços de APS no manejo de adultos usuários de crack. Entretanto, não foram encontrados artigos do contexto da APS para serem incluídos, e, mesmo ampliando as buscas em maior número de bases de dados bibliográficos, o número total de artigos foi pequeno.

Tabela 1. Avaliação metodológica dos seis estudos observacionais sobre manejo do crack pelo instrumento de Wales, Porto Alegre, 2013.

\begin{tabular}{|c|c|c|c|c|c|c|c|c|c|c|c|c|c|c|c|}
\hline Ref & Autor/ano & 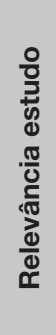 & 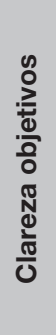 & $\begin{array}{l}\frac{\pi}{0} \\
\frac{\pi}{2} \\
\frac{0}{0} \\
\frac{0}{0} \\
\frac{\pi}{2} \\
\frac{\pi}{0} \\
\frac{0}{0} \\
\frac{0}{0} \\
\frac{0}{0}\end{array}$ & 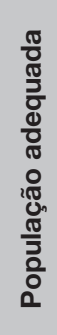 & 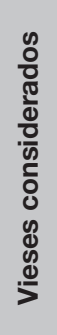 & 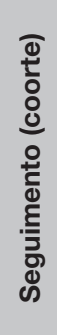 & $\begin{array}{l}0 \\
\frac{0}{0} \\
0 \\
\frac{0}{\pi} \\
0 \\
0 \\
0 \\
0 \\
0 \\
\frac{0}{\pi} \\
\frac{\pi}{0}\end{array}$ & 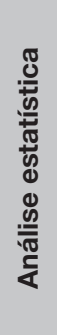 & 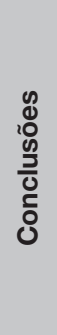 & \begin{tabular}{l}
$\frac{\pi}{5}$ \\
$\frac{5}{0}$ \\
\multirow{2}{x}{} \\
0 \\
$\frac{0}{0}$ \\
$\frac{\pi}{0}$ \\
$\frac{0}{\pi}$ \\
$>$
\end{tabular} & 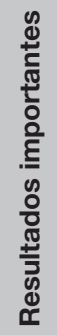 & 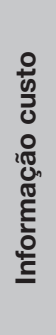 & $\begin{array}{l}\geq \\
\frac{\pi}{0} \\
\frac{\pi}{0} \\
\frac{0}{0} \\
\frac{0}{0} \\
\frac{\pi}{\pi} \\
\frac{\pi}{0}\end{array}$ & $\begin{array}{l}\bar{\pi} \\
\text { 음 }\end{array}$ \\
\hline 23 & Araujo et al., 2010 & $S$ & $S$ & $\mathrm{~N}$ & 0 & 0 & 0 & $\mathrm{~N}$ & $\mathrm{~N}$ & $S$ & $S$ & $\mathrm{~N}$ & $\mathrm{~N}$ & $\mathrm{~N}$ & 4 \\
\hline 36 & Zule et al., 2003 & $\mathrm{~S}$ & $\mathrm{~S}$ & $\mathrm{~S}$ & 0 & $\mathrm{~S}$ & 0 & $\mathrm{~S}$ & $\mathrm{~S}$ & $\mathrm{~S}$ & $\mathrm{~S}$ & $\mathrm{~S}$ & $\mathrm{~N}$ & $\mathrm{~S}$ & 10 \\
\hline 31 & Marsden et al., 2009 & $\mathrm{~S}$ & S & $\mathrm{N}$ & $\mathrm{N}$ & $\mathrm{N}$ & $\mathrm{N}$ & S & S & $\mathrm{S}$ & $\mathrm{s}$ & $\mathrm{S}$ & $\mathrm{N}$ & $\mathrm{S}$ & 8 \\
\hline 27 & Harocopos et al., 2003 & $\mathrm{~S}$ & $\mathrm{~S}$ & $\mathrm{~N}$ & $\mathrm{~N}$ & $\mathrm{~N}$ & $\mathrm{~S}$ & $\mathrm{~S}$ & $\mathrm{~N}$ & S & $N$ & $\mathrm{~N}$ & $\mathrm{~N}$ & $\mathrm{~S}$ & 6 \\
\hline 26 & Dias et al., 2011 & $\mathrm{~S}$ & $\mathrm{~S}$ & $\mathrm{~N}$ & $\mathrm{~N}$ & $\mathrm{~N}$ & $\mathrm{~S}$ & $\mathrm{~S}$ & $\mathrm{~S}$ & $\mathrm{~S}$ & $\mathrm{~S}$ & $\mathrm{~S}$ & $\mathrm{~N}$ & $\mathrm{~S}$ & 9 \\
\hline
\end{tabular}

$\mathrm{S}=\operatorname{sim} ; \mathrm{N}=$ não; 0 = não se aplica. 
Tabela 2. Avaliação metodológica dos 10 estudos com classificação do nível de evidência segundo o Sistema GRADE, Porto Alegre, 2013

\begin{tabular}{|c|c|c|c|c|c|c|c|c|c|}
\hline 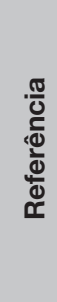 & 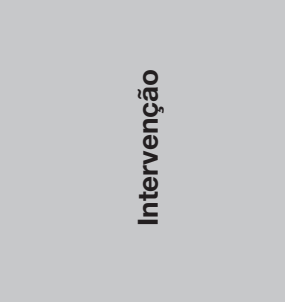 & $\begin{array}{l}\frac{?}{0} \\
\frac{\Phi}{5} \\
\text { Q }\end{array}$ & 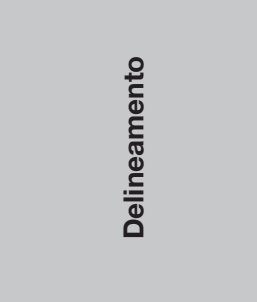 & 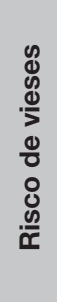 & $\begin{array}{l}\frac{0}{0} \\
\frac{\pi}{0} \\
\frac{0}{0} \\
\frac{c}{0} \\
\frac{0}{0} \\
\frac{0}{0} \\
\frac{0}{1}\end{array}$ & 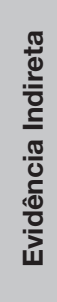 & 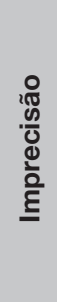 & 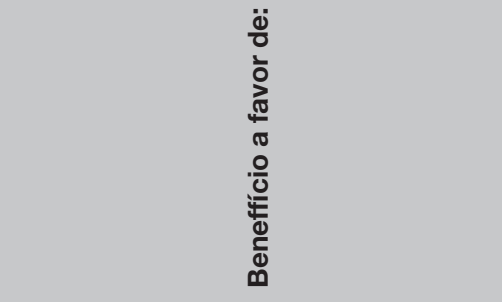 & $\begin{array}{l}\frac{\pi}{0} \\
\frac{c}{d} \\
\frac{20}{2} \\
\frac{0}{0} \\
\frac{0}{0} \\
\frac{2}{2}\end{array}$ \\
\hline 35 & $\begin{array}{l}\text { Relaxamento } \\
\text { respiratório }\end{array}$ & $\begin{array}{l}\text { Avaliar ansiedade } \\
\text { e craving }\end{array}$ & $\begin{array}{l}\text { Ensaio clínico } \\
\text { quase-experimen- } \\
\text { tal Tipo antes e } \\
\text { depois }\end{array}$ & -1 & nsa & -1 & -1 & $\begin{array}{l}\text { Exercícios respiratórios sobre } \\
\text { craving e ansiedade }\end{array}$ & D \\
\hline 32 & $\begin{array}{c}\text { Programa } \\
\text { multifacetado }\end{array}$ & $\begin{array}{l}\text { Uso de drogas } \\
\text { (crack e outras), } \\
\text { sexo de risco, sin- } \\
\text { tomas mentais, ati- } \\
\text { vidades criminais }\end{array}$ & $\begin{array}{l}\text { Ensaio clínico do } \\
\text { tipo quase-experi- } \\
\text { mental, tipo antes } \\
\text { e depois }\end{array}$ & -1 & nsa & -1 & -1 & $\begin{array}{c}\text { Programa multifacetado sobre } \\
\text { dias de uso de drogas, atividades } \\
\text { criminais }\end{array}$ & $D$ \\
\hline 34 & $\begin{array}{l}\text { Intervenção pré } \\
\text { e pró-tratamento, } \\
\text { multifacetada, } \\
\text { técnica motivacio- } \\
\text { nal, etc. }\end{array}$ & $\begin{array}{l}\text { Início de } \\
\text { tratamento }\end{array}$ & ecr & 0 & nsa & 1 & -1 & $\begin{array}{l}\text { Desfechos secundários com odds } \\
\text { pequenas e grandes intervalos de } \\
\text { confiança, sem efeito para desfecho } \\
\text { primário (começar tratamento) }\end{array}$ & C \\
\hline 33 & $\begin{array}{l}\text { Intervenção cogni- } \\
\text { tivo comportamen- } \\
\text { tal multifacetada }\end{array}$ & $\begin{array}{l}\text { Redução do con- } \\
\text { sumo de crack, } \\
\text { desfechos secun- } \\
\text { dários: compor- } \\
\text { tamento de risco } \\
\text { sexual/HIV }\end{array}$ & ecr & 0 & nsa & 1 & -1 & $\begin{array}{l}\text { Adequação de intervenções } \\
\text { cognitivocomportamentais com } \\
\text { aspectos culturais e de gênero sem } \\
\text { efeito sobre desfecho principal, com } \\
\text { efeito pequeno sobre desfechos } \\
\text { secundários }\end{array}$ & $B$ \\
\hline 30 & $\begin{array}{l}\text { Intervenção } \\
\text { comportamental }\end{array}$ & $\begin{array}{l}\text { Adesão a HAART, } \\
\text { consumo de crack } \\
\text { é secundário }\end{array}$ & ecr & 0 & nsa & -1 & -1 & $\begin{array}{l}\text { Efeito pequeno sobre consumo de } \\
\text { crack }\end{array}$ & C \\
\hline 29 & $\begin{array}{l}\text { Intervenção } \\
\text { comportamental } \\
\text { multifacetada }\end{array}$ & $\begin{array}{l}\text { Primário: consumo } \\
\text { de crack }\end{array}$ & ecr & 0 & nsa & -1 & -1 & $\begin{array}{l}\text { Sem efeito sobre o consumo, efeito } \\
\text { pequeno sobre condições de vida }\end{array}$ & C \\
\hline
\end{tabular}

Qualidade da evidência - $\mathrm{A}$ = alta; $\mathrm{B}$ = moderada; $\mathrm{C}$ = baixa; e $\mathrm{D}$ = muito baixa.

Além dessa limitação, a definição pelo período de busca dentro da última década, embora tenha embasamento em alguns referenciais que orientam a atualização para novas evidências nos últimos cinco ou até dez anos, ${ }^{7}$ não propiciou número maior de estudos nem forneceu informações que fizessem diferença em relação às publicações anteriores. 
Os conteúdos dos artigos observacionais foram muito distintos, tendo cada qual um foco diferente: avaliar estratégias para manejo de fissura, avaliar motivação para tratamento, mostrar "efetividade" de tratamentos em comunidade terapêutica, identificar formas de intervenção, correlacionar consumo e histórico de tratamento anterior, e avaliar intervenções breves, ou seja, enfoques distintos, o que impede a constituição de um conjunto mais robusto de evidências.

Os outros estudos mostraram intervenções heterogêneas (farmacológicas, terapia cognitiva, case manager, programa de redução de danos e outros) e, desta forma, impediram a realização de uma meta-análise.

Contudo, os achados apontam que diversas estratégias podem ser adaptadas à APS e a escolha de pesquisas em outras realidades não restrigiu a observação sobre as intervenções e/ou resultados apresentados. Zule et al., ${ }^{36}$ por exemplo, referem que a entrada ao tratamento e retenção pode ser facilitada pelo pré-tratamento e tratamento auxiliar. Esse resultado poderia ser lincado com a necessidade de fortalecer a rede integrada de saúde, com abordagens auxiliares e pré-tratamento contemplados nos cuidados primários. Já o artigo de Marsden et al., ${ }^{31}$ refere que os primeiros 6 meses de tratamento farmacológico ou psicológico estão associados com a redução da heroína e cocaína. Aqui, faz-se necessário lembrar que um dos atributos da APS é o acesso facilitado, e, unindo-se a isso, Kessler e Pechansky1 lembram que os casos que não têm fácil acesso ao sistema de saúde ou não têm apoio externo costumam ter baixos índices de recuperação. Logo, a ação de uma equipe de saúde com coordenação do cuidado pode fazer toda a diferença. No resultado da pesquisa de Alves e Araújo, ${ }^{37}$ foi encontrada diminuição significativa no craving e sintomas de ansiedade a partir dos jogos cooperativos, assim, técnicas comportamentais e com redução de sintomas podem ser ferramentas legítimas a serem realizadas no nível primário de atenção. No artigo de Sterk et al., ${ }^{33}$ o programa de redução de danos foi estatisticamente significante para reduzir o consumo de crack.

\section{Conclusões}

Apesar dos artigos não apresentarem evidências de alta qualidade e recomendações favoráveis que auxiliem a definir uma proposta baseada em evidências ao contexto da APS, muitos deles contribuíram com informações a respeito das experiências de abordagem em outros contextos. O estudo possibilitou aprofundar o conhecimento sobre o assunto, integrar resultados, avaliar as implicações para a prática e possibilitou rever as evidências científicas que permanecem como as atuais, assim como, entrar em contato com a opinião de diversos especialistas.

Segundo Kessler e Pechansky, ${ }^{1}$ está claro para a comunidade científica e leiga brasileira que o crack é uma droga de grande impacto e de difícil tratamento. Os autores afirmam que para a abordagem deste problema é necessário um enfoque com características biopsicossociais e um modelo de atenção multidisciplinar; mas ressaltam que a estratégia que parece demonstrar maior resultado contempla o tratamento com uma internação inicial em ambiente psiquiátrico localizado em hospital geral, e tem continuidade com um modelo de atendimento baseado em comunidades terapêuticas fechadas ou com alto grau de intensidade de tratamento, também por longos períodos, variando de seis meses a um ano.

O uso de psicofármacos costuma auxiliar, principalmente, em situações nas quais existam outras comorbidades psiquiátricas, pois não há medicação específica para o uso de crack.1,20 
Ribeiro e Laranjeira ${ }^{20}$ afirmam que não há um serviço melhor do que o outro, e sim pacientes mais indicados para determinado serviço. Entretanto, é reconhecido que o perfil do usuário de crack está associado com padrões de consumo da droga mais pesado, envolvimento com contravenções, complicações psiquiátricas e, com mais frequência, altos índices de recaídas com a droga. Desta forma, os usuários que apresentam padrão severo de comportamento relacionados ao consumo, exigirão medidas intensas capazes de produzir resultados benéficos.

As experiências em manejo de adultos usuários de crack no contexto da Atenção Primária em outros países não podem ser diretamente replicadas no Brasil, mas muitas diretrizes lá convencionadas podem sugerir orientações e dar sentido aqui, se adaptadas à nossa cultura e ao nosso modelo de organização dos serviços de saúde.

Os transtornos por uso de substâncias são problemas crônicos com sérios componentes de ordem biológica, psicológica e social, que trazem consequências de mesma ordem. Portanto, a solução não é exclusiva do setor da saúde e passa por um grande desafio em compor esforços que diminuam ou eliminem impasses.

Os serviços de saúde devem estar estruturados de forma a ocorrerem níveis de atendimento, e, os papeis dos profissionais da saúde, no tratamento da dependência química, devem estar normatizados. ${ }^{20}$ O manejo de adultos usuários de crack deve ter início na APS. ${ }^{17,40,41}$

Existe uma característica exclusiva da APS, que é a responsabilidade sobre sua área geográfica de abrangência, e que, independentemente da solicitação de ajuda das pessoas, cabe aos profissionais detectar problemas, ou estar atentos às possibilidades dos diversos problemas. ${ }^{42}$ Muitos usuários de drogas não vão fazer menção ao uso e tampouco solicitar ajuda, e as famílias, muitas vezes, nem percebem os sinais. Sendo assim, é o profissional que oferece ajuda e torna-se responsável por dar início ao processo terapêutico. Nesta situação, a prática de explicitar que a Unidade de Saúde recebe pessoas com dificuldades com drogas é uma atitude simples, mas que pode facilitar o acesso e definir a porta de entrada. ${ }^{17,43,44}$ Os que solicitam ajuda espontaneamente devem ser conduzidos à consulta de atendimento do dia para serem avaliados clinicamente e ter o perfil de usuário definido. O pronto atendimento é fundamental pelo alto índice de falhas às marcações de consultas. ${ }^{14,17,40,41,44}$

A avaliação clínica e o risco social também poderão definir o nível de atenção e tudo isto pode ser realizado na Atenção Primária.

A APS, por seguir a ordem da complexidade, contextualiza, trata o conjunto, generaliza e, ao mesmo tempo, trata o indivíduo, reconhece o singular. Desta forma, para operacionalizar e guiar as ações, é fundamental lembrar os atributos essenciais da APS - a acessibilidade, a longitudinalidade, a integralidade e a coordenação do cuidado. Assim sendo, foram encontradas na literatura diversas ações que são atribuições essenciais da APS, como:

1. Facilitar o acesso; ${ }^{17,40,41,43,44}$

2. Definir o perfil do usuário; ${ }^{17,41}$

3. Identificar problemas clínicos e sociais; ${ }^{17,20,40,41,43,44}$

4. Avaliar riscos e contatar setores específicos se necessário; ${ }^{17,20,40,41}$

5. Planejar o tratamento conjuntamente com a família e o indivíduo; ${ }^{17,43}$ 
6. Organizar o cuidado, incluindo outros profissionais; $;^{17,43,44}$

7. Traçar rede de apoio; ${ }^{17,43,44}$

8. Sugerir processos psicoterapêuticos: ${ }^{17,40,41,43,44}$

9. Auxiliar o processo de inclusão social; ${ }^{17,40,41,43,44}$

10. Acompanhar longitudinalmente. ${ }^{41,44}$

AAPS é a porta de entrada da maioria dos usuários de drogas. Mas a maioria das unidades não é capaz de fornecer a gama completa de tratamentos possíveis. Portanto, é fundamental saber os recursos relevantes locais e para onde as pessoas podem ser encaminhadas rapidamente. ${ }^{17}$

O sistema de notificação de todos os usuários e o contato com o CAPS, assim como a avaliação conjunta, quando necessária, deveria ser a rotina de uma rede integrada.

O uso de medicações deve ser individualizado e, já que as abordagens psicoterápicas são aquelas que concentram maior evidência, as intervenções, como a abordagem de família, a terapia cognitiva comportamental, a entrevista motivacional e a prevenção de recaídas, devem estar disponíveis para serem acessadas. 17,40,41,43,44 Portanto, faz-se necessário o direcionamento de recursos neste nível de atenção para que se possam realizar treinamentos específicos.

\section{Referências}

1. Kessler F, Pechansky F. Uma visão psiquiátrica sobre o fenômeno do crack na atualidade. Rev Psiquiatr Rio Gd Sul. 2008;30(2):96-8. DOI: http://dx.doi.org/10.1590/S0101-81082008000300003

2. Conselho Federal de Medicina. Diretrizes gerais médicas para assistência integral ao crack [Internet]. Brasília: CFM; 2011 [acesso 2013 Mar 29]. Disponível em: http://www.sbp.com.br/pdfs/diretrizes-medicas-integral-crack-cfm.pdf

3. Cavalcante T, Fundação Oswaldo Cruz. Maior pesquisa sobre crack já feita no mundo mostra o perfil do consumo no Brasil [Internet]. 2013 [acesso 2013 Dec 5]. Disponível em: http://portal.fiocruz.br/pt-br/content/maior-pesquisa-sobre-crack-j\%C3\%A1-feita-no-mundo-mostra-o-perfil-do-consumo-no-brasil

4. Brasil. Assessoria de Comunicação do Ministério da Justiça (BR). Governo vai investir R\$ 4 bilhões em ações para enfrentar o crack (07/12/2011) [Internet]. 2011 [cited 2013 mai 12]. Available from: http://www.obid.senad.gov.br/portais/OBID/ conteudo/web/noticia/ler_noticia.php?id_noticia $=105460$

5. Associação Brasileira de Psiquiatria. Abuso e dependência: crack. Rev Assoc Med Bras. 2012;58(2):141-53. PMID: 22569607. DOI: http://dx.doi.org/10.1590/S0104-42302012000200008

6. University of Oxford. P I C O: Formulate an answerable question [Internet]. 2013 [cited 2013 jul 20]. Available from: http:// learntech.physiol.ox.ac.uk/cochrane_tutorial/cochlibd0e84.php.

7. Weightman AL, K. MM, Sander L, Turley RL. Appendix 8: Questions to assist with the critical appraisal of an observational study eg cohort, case-control, crossectional. 2004 [cited 9 jul 2013]. In: Project methodology 5 [Internet]. Cardiff: Information Services UWCM, [cited 9 jul 2013]. Available from: http://hebw.cf.ac.uk/projectmethod/Project\%20Methodology\%205.pdf

8. Guyatt G, Hayward R, Richardson WS, Green L, Wilson M, Sinclair J, et al. Moving from evidence to action. 2002 [cited 10 ago 2013]. In: Users' guides to the medical literature: a manual for evidence-based clinical practice [Internet]. New York: McGraw Hill, [cited 10 ago 2013]. Available from: http://medicine.ucsf.edu/education/resed/articles/jama1_moving.pdf.

9. Araujo RB, Balbinot AD, Castro MGT, Rocha MR, Miguel SRPS, Cohen M, et al. Tratamento de exposição a estímulos e treinamento de habilidades como coadjuvantes no manejo do craving em um dependente de crack. Trends Psychiatry Psychoter. 2011;33(3):181-8. DOI: http://dx.doi.org/10.1590/S2237-60892011000300008

10. Araujo RB, Castro MGT, Pedroso RS, Santos PL, Leite L, Rocha MR, et al. Validação psicométrica do Cocaine Craving Questionnaire-Brief - Versão Brasileira Adaptada para o Crack para dependentes hospitalizados. J Bras Psiquiatr. 2011;60(4):233-9. DOI: http://dx.doi.org/10.1590/S0047-20852011000400001 
11. Bastos FI. Crack no Brasil: uma emergência de saúde. Cad Saúde Pública. 2012;28(6):1016-7. PMID: 22666806. DOI: http://dx.doi.org/10.1590/S0102-311X2012000600001

12. Bernardo WM, Marques ACPR. Atualização em abuso e dependência: crack. Rev Assoc Med Bras. 2012;58(3):287. DOI: http://dx.doi.org/10.1590/S0104-42302012000300006

13. Brown EJ, Hill MA, Giroux SA. "A 28-day program ain't helping the crack smoker"--perceptions of effective drug abuse prevention interventions by north central Florida African Americans who use cocaine. J Rural Health. 2004;20(3):286-95. PMID:15298105. DOI: http://dx.doi.org/10.1111/j.1748-0361.2004.tb00041.x

14. Chaves TV, Sanchez ZM, Ribeiro LA, Nappo SA. Fissura por crack: comportamentos e estratégias de controle de usuários e ex-usuários. Rev Saúde Pública. 2011;45(6):1168-75. PMID:21894428. DOI: http://dx.doi.org/10.1590/S003489102011005000066

15. D'Alberto A. Auricular acupuncture in the treatment of cocaine/crack abuse: a review of the efficacy, the use of the National Acupuncture Detoxification Association protocol, and the selection of sham points. J Altern Complement Med. 2004;10(6):985-1000. PMID:15673993. DOI: http://dx.doi.org/10.1089/acm.2004.10.985

16. Domanico A, Malta M. Implementation of harm reduction toward crack users in Brazil: barriers and achievements. Subst Use Misuse. 2012;47(5):535-46. PMID:22428821. DOI: http://dx.doi.org/10.3109/10826084.2012.644170

17. Ford C. Guidance for working with cocaine and crack users in primary care [Internet]. London: Royal College of General Practitioners; 2004 [cited 29 mar 2013]. Available from: http://www.drugsandalcohol.ie/13634/

18. Kolling NM, Petry M, Melo WV. Outras abordagens no tratamento da dependência do crack. Rev Bras Ter Cogn. 2011;7(1):714.

19. Oliveira EN, Lira TQ, Ferreira AGN, Araujo JVB, Nogueira NF, Marinho MP, et al. Aspectos relacionados ao tratamento de usuários de crack e álcool em um serviço de saúde mental. Rev Tendên da Enferm Profis. 2012;4(2):687-91.

20. Ribeiro M, Laranjeira R. O tratamento do usuário do crack. São Paulo: Casa Leitura Médica; 2012.

21. Rodrigues DS, Backes DS, Freitas HMB, Zamberlan C, Gelhen MH, Colomé JS. Conhecimentos produzidos acerca do crack: uma incursão nas dissertações e teses brasileiras. Cienc Saúde Coletiva. 2012;17(5):1247-58. DOI: http://dx.doi. org/10.1590/S1413-81232012000500018

22. Vorspan F, Bellais L, Keijzer L, Lépine JP. An open-label study of aripiprazole in nonschizophrenic crack-dependent patients. J Clin Psychopharmacol. 2008;28(5):570-2. PMID:18794657. DOI: http://dx.doi.org/10.1097/JCP.0b013e3181858311

23. Araujo RB, Pansard M, Boeira BU, Rocha NS. As estratégias de coping para o manejo da fissura de dependentes de crack. Rev HCPA. 2010;30(1):36-42.

24. Campbell J, Nickel EJ, Penick EC, Wallace D, Gabrielli WF, Rowe C, et al. Comparison of desipramine or carbamazepine to placebo for crack cocaine-dependent patients. Am J Addict. 2003;12(2):122-36. PMID:12746087. DOI: http://dx.doi. org/10.1111/j.1521-0391.2003.tb00610.x

25. Corsi KF, Rinehart DJ, Kwiatkowski CF, Booth RE. Case management outcomes for women who use crack. J Evid Based Soc Work. 2010;7(1):30-40. PMID:20178023. DOI: http://dx.doi.org/10.1080/15433710903175858

26. Dias AC, Araújo MR, Laranjeira R. Evolução do consumo de crack em coorte com histórico de tratamento. Rev Saúde Pública. 2011;45(5):938-48. PMID:21808833. DOI: http://dx.doi.org/10.1590/S0034-89102011005000049

27. Harocopos A, Dennis D, Turnbull PJ, Parsons J, Hough M. On the rocks: a follow-up study of crack users in London [Internet]. London: Criminal Policy Research Unit; 2003 [cited 16 jun 2013]. Available from: http://www.drugsandalcohol. ie/5446/1/1716-1637.pdf

28. Hart CL, Haney M, Vosburg SK, Rubin E, Foltin RW. Gabapentin does not reduce smoked cocaine self-administration: employment of a novel self-administration procedure. Behav Pharmacol. 2007;18(1):71-5. PMID:17218799. DOI:http:// dx.doi.org/10.1097/FBP.0b013e328014139d

29. Henskens R, Garretsen H, Bongers I, Van Dijk A, Sturmans F. Effectiveness of an outreach treatment program for inner city crack abusers: compliance, outcome, and client satisfaction. Subst Use Misuse. 2008;43(10):1464-75. PMID:18615321. DOI: http://dx.doi.org/10.1080/10826080500391613 
30. Ingersoll KS, Farrell-Carnahan L, Cohen-Filipic J, Heckman CJ, Ceperich SD, Hettema J, et al. A pilot randomized clinical trial of two medication adherence and drug use interventions for HIV + crack cocaine users. Drug Alcohol Depend. 2011;116(1-3):177-87. PMID: 21306837 DOI: http://dx.doi.org/10.1016/j.drugalcdep.2010.12.016

31. Marsden J, Eastwood B, Bradbury C, Dale-Perera A, Farrell M, Hammond P, et al. Effectiveness of community treatments for heroin and crack cocaine addiction in England: a prospective, in-treatment cohort study. Lancet. 2009;374(9697):126270. DOI: http://dx.doi.org/10.1016/S0140-6736(09)61420-3

32. Okpaku S, Macmaster SA, Dennie S, Tolliver D, Cooper RL, Rasch RF. Preliminary outcomes of a model program for increasing treatment access for African American women who use crack cocaine and are at risk for contracting HIV. J Evid Based Soc Work. 2010;7(1):41-57. PMID: 20178024. DOI: http://dx.doi.org/10.1080/15433710903175874

33. Sterk CE, Theall KP, Elifson KW. Effectiveness of a risk reduction intervention among African American women who use crack cocaine. AIDS Educ Prev. 2003;15(1):15-32. PMID:12627741. DOI: http://dx.doi.org/10.1521/aeap.15.1.15.23843

34. Wechsberg WM, Zule WA, Riehman KS, Luseno WK, Lam WK. African-American crack abusers and drug treatment initiation: barriers and effects of a pretreatment intervention. Subst Abuse Treat Prev Policy. 2007;2:10. PMID:17394653 PMCid:PMC1847815. DOI: http://dx.doi.org/10.1186/1747-597X-2-10

35. Zeni TC, Araujo RB. O relaxamento respiratório no manejo do craving e dos sintomas de ansiedade em dependentes de crack. Rev Psiquiatr Rio Gd Sul. 2009;31(2):116-9. DOI: http://dx.doi.org/10.1590/S0101-81082009000200006

36. Zule WA, Lam WK, Wechsberg WM. Treatment readiness among out-of-treatment African-American crack users. J Psychoactive Drugs. 2003;35(4):503-10. PMID:14986880. DOI: http://dx.doi.org/10.1080/02791072.2003.10400498

37. Alves GSL, Araujo RB. A utilização dos jogos cooperativos no tratamento de dependentes de crack internados em uma unidade de desintoxicação. Rev Bras Med Esporte. 2012;18(2):77-80. DOI: http://dx.doi.org/10.1590/S151786922012000200002

38. Bisch NK, Benchaya MC, Signor L, Moleda HMR, Ferigolo M, Andrade TMR, et al. Aconselhamento telefônico para jovens usuários de crack. Rev Gaúcha Enferm. 2011;32(1):31-9. PMID:21888200. DOI: http://dx.doi.org/10.1590/S198314472011000100004

39. Brasil. Ministério da Saúde. Diretrizes metodológicas: elaboração de pareceres técnico-científicos. 3a ed. Brasília: Ministério da Saúde; 2011.

40. Greater London Authority. The GLADA crack cocaine strategy 2005-08. London: Greater London Authority; 2004.

41. National Treatment Agency for Substance Misuse. Models of care for treatment of adult drug misusers: Update 2006 [Internet]. London: NHS; 2006 [cited 10 ago 2013]. Available from: http://www.nta.nhs.uk/uploads/nta_modelsofcare_update_2006_moc3.pdf.

42. Starfield B. Atenção primária: equilíbrio entre necessidades de saúde, serviços e tecnologia. Brasília: UNESCO; 2002.

43. Connolly J, Foran S, Donovan AM, Carew AM, Long J. Crack cocaine in the Dublin region: an evidence base for a Dublin crack cocaine strategy [Internet]. Dublin: Health Research Board; 2008 [cited 29 jul 2013]. Available from: http://www. hrb. ie/uploads/tx_hrbpublications/HRB_Research_Series_6.pdf

44. U.S. Department of Health and Human Services, National Institutes of Health, National Institute on Drug Abuse. Principles of drug addiction treatment: a research-based guide [Internet]. Washington: $\mathrm{NIH} ; 1999$ [cited 13 mar 2013]. Available from: http://www.drugabuse.gov/sites/default/files/podat_1.pdf 